\title{
Space-track modulation and coding for high density aerial vehicle downlink networks with free space optical and visible light communications
}

\author{
Burhan GÜLBAHAR*(i) \\ Department of Electrical and Electronics Engineering, Faculty of Engineering, Özyeğin University, İstanbul, Turkey
}

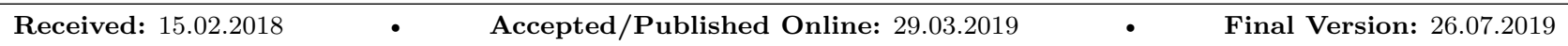

\begin{abstract}
Aerial vehicles (AVs) have challenges in terms of realizing low complexity and wide coverage area wireless communications architectures, especially for crowded or high density groups of AVs with state-of-the-art free space optical (FSO) and radio frequency (RF) based system designs. FSO architectures generally target point-to-point high gain directional links while requiring strict acquisition and tracking due to the narrow beam width of laser transmitters with challenges of vibration, turbulence, misalignment, atmospheric absorption, scattering, and fading. In this article, a novel multiuser free space optical system design of modulation and coding denoted by space-track modulation and coding (STMC) is proposed for crowded downlink communications. STMC does not require high accuracy tracking and costly system components by utilizing large beam divergence angles, more transmitter units with smaller aperture sizes and weights, and large spatial regions to receive modulated data at AV tracks. In addition, the spatio-optical wireless caching problem is introduced for AV networks while defining STMC problem information theoretically. STMC combines track based multiuser modulation and coding, with geographical and positional data for low data rate broadcasting applications with wide area coverage. Numerical simulations provide tens of bit/s data rates for thousands of kilometers of coverage area while simultaneously serving a significantly larger number of AVs. Finally, future works and open issues are discussed. The novel modulation and coding mechanisms are promising future applications for both high altitude AVs and low altitude drone networks with low complexity hardware for multiuser low data rate communications.
\end{abstract}

Key words: Space-track modulation and coding, aerial vehicle, satellite, high altitude platform, airplane, drone, free space optic, visible light communications

\section{Introduction}

Wireless communications networking for unmanned aerial vehicles (UAVs), high altitude platforms (HAPs), balloons, aircrafts and drones are important for various services such as commercial data and multimedia services to airplanes, remote sensing, geographical mapping, astronomical observations, military tasks, surveillance, and quantum cryptography networks [1]. On the other hand, drones promise additional applications such as environmental monitoring, Internet delivery, and goods transportation [2]. Free space optical (FSO) architectures among satellites, ground stations, and aerial vehicles (AVs) are promising as alternative platforms to radio frequency $(\mathrm{RF})$ communications with high data transmission capability and long range communications reaching thousands of kilometers, no interference problems as in RF channels, smaller antenna sizes and transmit powers, and huge optical bandwidths $[1,3-5]$. Bidirectional free space optical link between a geostationary (GEO) satellite and an aircraft is experimentally shown to achieve tens of Mbit/s data rates for the range of approximately

*Correspondence: burhan.gulbahar@ozyegin.edu.tr 
40,000 km [6]. Similarly, low-earth orbit (LEO) satellite to HAP communication links achieve several Gbit/s data communications with ranges of hundreds to thousands kilometers, utilizing laser transmission powers of tens of watts [1]. On the other hand, state-of-the-art satellite based FSO links require strict acquisition and tracking due to the narrow beam width of laser transmitters [3]. Pointing loss occurs due to satellite vibration, platform jitter, and atmospheric turbulence induced beam wandering. Furthermore, turbulence induced beam wandering, spreading, scintillation, beam divergence loss, misalignment, atmospheric absorption, scattering, and fading make the line-of-sight (LOS) FSO link more challenging, especially for multiple AV platforms $[1,3]$. Therefore, broadcasting mechanisms in channels between satellites and AVs are generally neglected in the recent literature. Moreover, in crowded networks with high density of AVs, the requirement for the same information content to be downloaded by a large number of AVs, e.g., a multimedia file, a packet, or control information, makes FSO links between a single transmitter and AV with high accuracy tracking more impractical. In this article, a simple system design of multiple AV downlink communications denoted by space-track modulation (STM) and coding (STMC) is proposed with coarse knowledge of vehicle tracks and without requiring accurate tracking or narrow beam dimensions [7]. Networks including crowded sets of AVs with varying velocities are targeted in this article such that tracking, optimizing data transmission, and broadcasting are very difficult. Besides that, STMC utilizes smaller transmitter aperture size with less weight of each modulation unit while allowing more units in the same area to create more STMC data spatial regions at receiver AV tracks.

The proposed architecture is also applicable for downlink channels of crowded swarms of drones. Drone networks are recently discussed with enabling technologies such as WiMax in [8] compared with other technologies such as Wi-Fi and ZigBee not satisfying the requirements of drone networks. Although the WiMax system proposes flexibility, high throughput, mobility, and large coverage, the problems of interference, synchronization, and air traffic regulation of crowded drone networks are emerging open issues [2]. On the other hand, it is highly challenging to operate the system at altitudes higher than $100 \mathrm{~km}$ and in areas where WiMax network infrastructure does not exist. Similar problems exist for cellular system based drone networks while satellite communications with drones have similar problems in terms of cost and complexity with FSO and RF based designs between satellites and AVs including HAPs and aircrafts [2, 9].

In this article, a novel FSO modulation and coding mechanism denoted by STMC is proposed for crowded networks of AVs including aircrafts, UAVs, HAPs, and drones. FSO links are obtained either with laser transmitters for long distance communications such as tens of thousands of kilometer ranges (by also including visible light communications (VLC) frequencies) or LED based VLC for shorter ranges, e.g., drone communications. Satellite transmitters are utilized for high altitude AV receivers with large sizes while balloons and HAPs function as transmitter sources for drone receiver networks in addition to satellite based transmitters. Large area beam spreading spatial regions are utilized along the tracks of AVs with the proposed STM mechanism and a global coding strategy achieves to broadcast data while AVs pass through their tracks. Large beam divergence angles result in long radius regions denoted by STM regions on the receiver tracks, i.e. modulated optical signal regions or color cells with large beam divergence angle at the receiver location. These regions provide transmission of data to the receiver with limited knowledge of AV tracking and without requiring high pointing accuracy or narrow beam angle. A cooperative broadcast coding mechanism is proposed where data links in STM regions are utilized for multiple AVs. In addition, wireless caching architectures denoted by spatiooptical wireless caching architectures are introduced by exploiting STM cells or regions as broadcast regions $[10,11]$. Moreover, energy of transmission is harvested from the sun as an energy efficient system design by using solar powered or solar pumped laser structures as thoroughly discussed in [12-14] while the design of harvesting 
units is out of scope of the article. They are efficient to operate Nd:YAG rod structures with tens to hundreds of watts CW laser power, enough to realize STMC system designs covering large area satellite networks [14].

The proposed architecture as an alternative to long range RF communications and existing FSO systems has important advantages as summarized in the following list $[3,15]$ :

- VLC offers higher data rates with much larger available bandwidths, i.e. thousands of times larger than $\mathrm{RF}$ bandwidth, in the $400 \mathrm{THz}$ to $800 \mathrm{THz}$ frequency range.

- Optical antennas are more compact and power efficient compared with RF, which is especially important for aerial networks due to size and power constraints. Beam divergence is proportional to $\lambda / D_{T}$, where $\lambda$ is the carrier wavelength and $D_{T}$ is the aperture diameter and varies inversely with antenna gain. RF wavelength is approximately a thousand times larger than optical wavelength, requiring a much larger RF antenna to achieve the same gain, e.g., typical sizes are $0.3 \mathrm{~m}$ and $1.5 \mathrm{~m}$ for optical and RF spacecraft antennas, respectively [3].

- For long range applications, RF beam divergence is significantly large compared with optical communications, making it less secure compared with VLC, while RF signals can penetrate through obstacles and can be detected with spectrum analyzers.

- The VLC frequency band is free without any license.

- Existing multiuser FSO architectures utilize independent component analysis (ICA) for blind source separation (BSS) problems in multipoint mobile systems with independent transmitters [16] and scanning systems by steering for acquisition and tracking $[17,18]$. However, in the proposed architecture, a novel form of multiuser communications combines space and track information with multiuser coding without requiring tracking and beam steering. Therefore, compared with the nontracking FSO system, it provides a novel multiuser modulation and coding technique as discussed in detail in Sections 4 and 5.

The novel contributions proposed in this article are given as follows:

1. A novel FSO modulation and coding method denoted by STMC is defined for downlink of crowded networks of multiple AVs, which can be used for a rich set of applications at varying altitudes and with varying velocities of transmitters and receivers by using a specifically designed system set-up.

2. STMC achieves multiuser data transmission to AVs without requiring complicated hardware, including high accuracy tracking and synchronization, narrow beam laser sources, and pointing. It provides a larger number of laser modulator units with smaller aperture sizes and weights for each unit.

3. Spatio-optical wireless caching is introduced for networks of AVs for broadcast based cooperation among $\mathrm{AVs}$ and downlink transmission of the same information content such as popular multimedia files, the same data packets, or broadcast control data.

4. STMC combines track based modulation with geographical and positional data by promising novel broadcast applications similar to the Global Positioning System (GPS) with wide area modulated color cells along the tracks of AVs.

5. The STMC problem is information theoretically defined as a fundamental model for future studies.

6. Future works and open issues of STMC system design are provided. 
The remainder of the paper is organized as follows. In Section 2, system modeling is presented, while the FSO channel model is described in Section 3. Networking architectures of the proposed STMC system are discussed in Section 4, while information theoretical modeling of the STMC architecture and definition of spatio-optical wireless caching are presented in Section 5. Numerical simulations and discussions are provided in Section 6. Open issues and challenges are discussed in Section 7, while the conclusions are given in Section 8.

\section{System model}

STMC system design includes a transmitter source located in the sky at a specified altitude and a receiver photodetector array on AVs. The transmitter is located on GEO and LEO satellites for large size AV networking, such as high velocity airplanes, HAPs, and balloons, while it can be located on another HAP or balloon for smaller size AV networking such as drone based architectures. GEO and LEO satellites are utilized in a cooperative networking scenario for the complete system design, while in this article utilization of a physical channel between a single transmitter and multiple receivers is analyzed in terms of the proposed novel STMC multiuser modulation and coding mechanisms. On the other hand, any aerial platform energy harvesting from solar resources and optimizing beam divergence angle to realize the targeted STM regions is valid for the proposed set-up. Therefore, the STMC system and information theoretical model in this article promise future utilization of STMC for crowded networks of AVs with varying altitudes and velocities of both transmitters and receivers in a rich set of applications.

A transmitter laser resource operating with various wavelengths including the visible light spectrum for VLC or infrared region for FSO communications is utilized. On the other hand, the proposed system set-up is

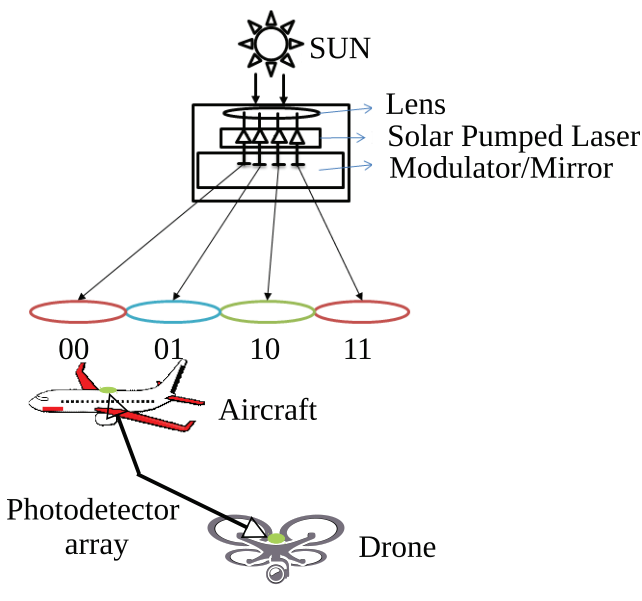

(a)

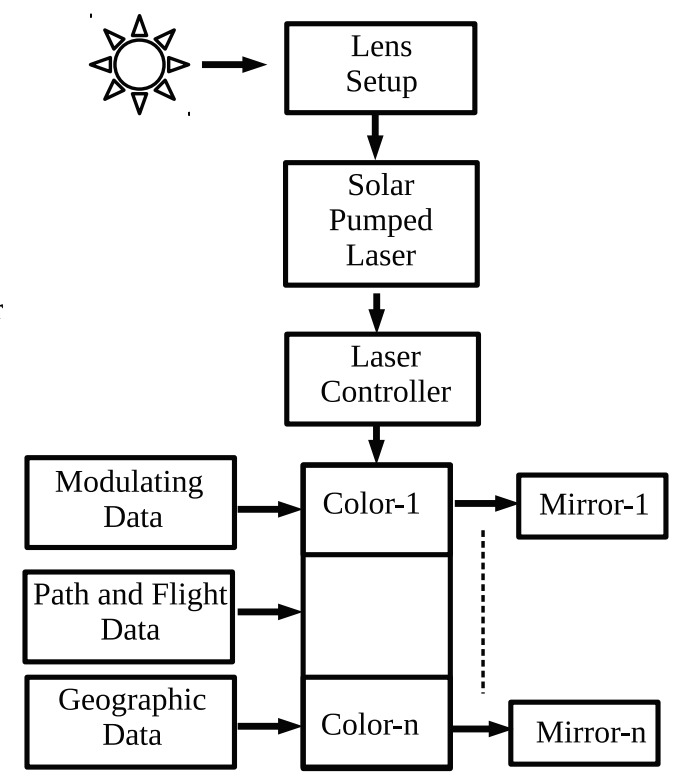

(b)

Figure 1. (a) STMC system design where the transmitter harvesting solar energy to feed laser sources creates modulated optical signal with large beam spread on the receiver photodetector arrays of AVs such as aircrafts and drones while utilizing an optical set-up including lenses and mirror units to collect and direct the light. STM regions have intensity and/or color modulated optical signals with a designed mapping between symbols and source data. (b) STMC system block diagram with capability to modulate multiple colors and to point out different STM regions by using mirror set-ups while modulation and coding block utilize geographical data and path and flight data of AVs. 
applicable for high power LED sources for VLC in smaller area networks, e.g., a drone network for hundreds of meters of range, with specially designed LED beaming set-up [19, 20]. The transmitter is not required to have an accurate tracking and beam pointing system design such that the data are spread along the track of AVs on large beam divergence angles as shown in Figures 1a and 1b, where the STMC system design and block diagram are shown, respectively. Coarse knowledge or estimation of AV tracks is utilized to realize an optimized encoding architecture. On the other hand, there is a trade-off between pointing accuracy and data capacity, as discussed in Section 3, such that high power resources are necessary to realize high data links with large beam divergence angles. However, in this article, downlink service for a crowded set of AVs is targeted such that high velocities and large numbers of AVs make it challenging to track and optimize data transmission for broadcast purposes. Furthermore, the same information content to be requested by a large number of AVs makes accurately tracked single FSO links more impractical.

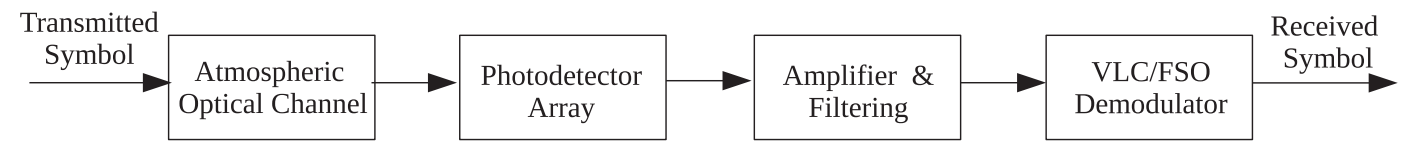

Figure 2. Receiver block diagram demodulating the optical signal received through atmospheric optical channel with a set of photodetector arrays, amplifying and filtering operations, and special demodulators designed for FSO and VLC systems.

The downlink receiver architecture as shown in Figure 2 includes a simple photodetector array without requiring tracking while the data are received along the tracks of AVs. The data are received along the large beam divergence angle regions while AVs are flying along their tracks or paths. This creates a time diversity for low data rate communications, e.g., keeping the modulated bit stream the same along STM regions for the duration of the passage of the AV. In addition, the proposed global encoding approach allows the data to be received along the path of the AV with a delayed decoding. There is a trade-off between delay and the received instantaneous data rate for the required information due to large beam divergence angle resulting in smaller received power. The analysis of the trade-off is an open issue and left as future work.

There are various sources of noise decreasing the sensitivity of the receiver to the received optical signal, including background noise from the atmosphere and sun, scattered light collected, and receiver based dark currents, shot noise, and thermal noise [15]. In this article, conventional receiver hardware in FSO satellite communications, i.e. photodetectors with intensifiers, mirrors, amplifiers, and filters for undesired noise sources, as shown in Figure 2, can be utilized, achieving tens of Mbit/s data rates with tens of watts transmission power [21-23]. These include various combinations of PIN photodiodes, avalanche photodiodes (APD), and photomultiplier tubes (PMTs) with high gain front ends while having disadvantages of higher weight and larger size [23]. On the other hand, beacon detectors such as charge-coupled devices (CCDs), charge injection devices (CIDs), and low complexity photodetectors with large field of view and without complicated amplifier structures target low data rate communications with higher beam divergence in the proposed architecture, especially for drone based shorter range aerial communication networks. Therefore, there is a trade-off between receiver complexity and the targeted data rate, which is in a lower regime in this article. Besides that, time diversity for low data rate communications and capability to serve multiple users simultaneously provide better sensitivity of the receiver to the external and internal effects, as discussed in Sections 4 and 6 .

The energy harvesting unit includes both solar panels exploiting infrared and visible light waves from the sun and also a solar powered laser infrastructure for laser based system set-up [12]. Similarly, the LED based 
set-up utilizes harvested energy to feed LED modulator resources. On the other hand, optical and electrical tasks including mirrors, filters, amplifiers, and data processing are performed with the harvested energy. A system design for GEO and LEO orbits is presented for ultra-violet (UV) laser systems harvesting tens of kilowatts of power used for laser and electrical system components in [13].

\section{FSO channel model}

Laser beam divergence angle $\theta$ is proportional to $\propto d_{f} \lambda / D_{T}$ in radians, where $\lambda$ is the carrier wavelength, $D_{T}$ is the aperture diameter, and $d_{f}$ is the divergence factor [1]. $d_{f}$ is taken as unity for simplicity without considering the effects of truncation and obscuration [24]. The beam radius at distance $L$ is given by simple trigonometric identity as follows:

$$
r_{j}=L \tan \left(\frac{\theta_{j}}{2}\right)
$$

Aircrafts pass through modulated symbols (including spatial, temporal, and optical frequency dimensions) with radius $r_{j}$ on their tracks encoded and optimized by the satellite transmitter. On the other hand, the received signal is affected by different parameters, including absorption depending on wavelength, Rayleigh and Mie scattering due to particles in the air, beam spreading, beam wander due to large scale turbulence, scintillation due to random index of refraction fluctuations, and phase-front distortions due to turbulent eddies [1]. In addition, dynamic weather conditions and background noise are other factors to be taken into account. The received power in the optical antenna aperture of the aircraft is modeled as follows with the assumption of ideal case of gain $[1,15,25]$ :

$$
P_{R} \approx P_{T} \frac{D_{R}^{2}}{L^{2} \theta^{2}} \gamma_{T} \gamma_{R} a_{T} a_{R}
$$

where $P_{T}$ is the transmit power, $D_{R}$ is the receiver diameter, $\gamma_{T}$ and $\gamma_{R}$ are respectively transmitter and receiver losses, and $a_{T}$ and $a_{R}$ are the losses due to atmosphere and pointing, respectively. In this article, a simplified system design is proposed while emphasizing the modulation and coding mechanism without getting into the details of channel modeling. FSO channel modeling has been thoroughly analyzed in various studies $[1,3,4]$. Effects of the channel on optimization of coding and modulation in STMC system architectures are left as future works. Next, the topology of the receivers in terms of the relation of their tracks with large radius STM regions is discussed.

\section{STMC networking architectures}

The receiver photodetector array on the AV passes through multiple spatially modulated areas with speed of $\vec{v}_{i}$ for $i \in[1, N]$ as shown in Figure 1a, where it is assumed that there is a set of $N$ vehicles in the STMC network. The time duration for each STM region $j$ where $j \in[1, M]$ is found as follows:

$$
\Delta t_{j, i}=\frac{2 r_{j}}{\vec{v}_{i}}
$$

In addition, a single region with radius $r_{j}$ includes multiple coded data transmission intervals as thoroughly modeled in Section 5. As the plane velocity is larger, the total number of coded transmission periods decreases in each region. On the other hand, the STM mechanism ensures the desired data transmission by optimizing the coded data in the regions. Besides that, neighbor STM cells are taken into account while an aircraft passes 
through a track since there is limited knowledge of the track in the transmitter. The large value of radius $r_{j}$ allows to transmit data without tracking as opposed to the difficulties encountered in precise tracking and data rate related problems in $\mathrm{RF}$ and optical laser communications counterparts.

Besides that, large values of radius $r_{j}$ decrease the received power in the AV while increasing $\Delta t_{j, i}$, allowing time diversity by simple repetitive coding in the same region. Although the individual capacity of each AV drops, the number of AVs in the broadcast channel increases by improving the capability for STMC system design without requiring accurate tracking.

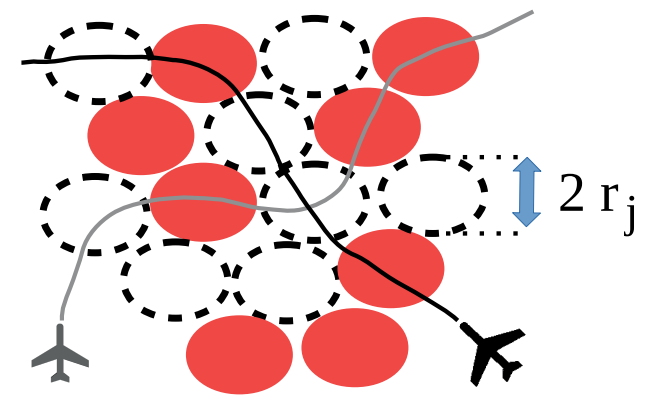

(a)

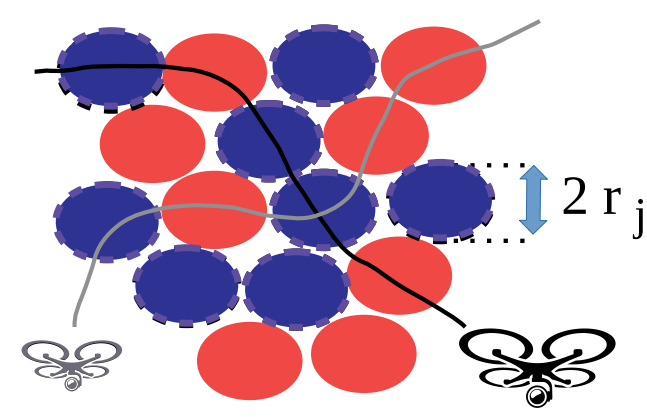

(c)

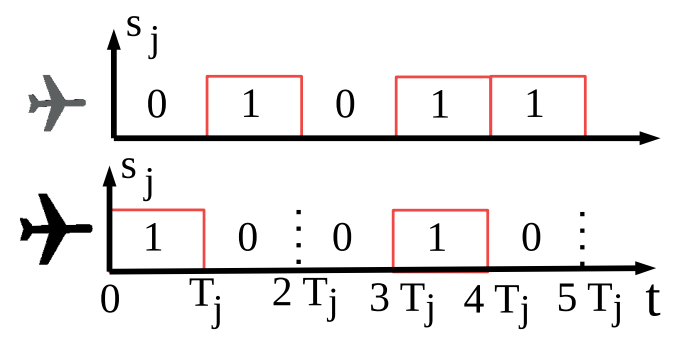

(b)

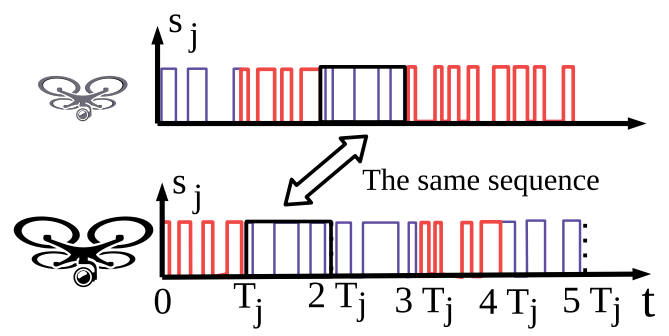

(d)

Figure 3. STMC example illustrating (a) low data rate ON-OFF keying transmission where each STM region encodes only a single bit and (b) utilizing the same region for coding the bit streams of multiple AVs. The same architecture is shown for drones with (c) high data rate multiple color ON-OFF keying transmission and (d) packets are used for different AVs in the same STM region.

\section{Information theoretical modeling of STMC and spatio-optical wireless caching}

STMC for the multireceiver broadcast data transmission problem is information theoretically modeled as follows:

- There are $N$ aerial vehicles such as aircrafts or drones requesting $N$ different independent messages denoted by $\left\{W_{1}, W_{2}, \ldots, W_{N}\right\}$.

- There is a set of $M$ unit regions denoted with $r_{j}$ for $j \in[1, M]$ with the ability to be distinctly modulated by the laser set-up in the transmitter, as shown in Figures 1 and 3. In Figures 3a and 3b, each STM region encodes only a single bit and the same region is utilized for coding multiple AVs, respectively, for low data rate applications. The similar scenarios for high data rate applications are shown in Figures $33 \mathrm{c}$ and $3 \mathrm{~d}$ for the drones. It is assumed that the regions can have different radius values changing with respect to the distance to the transmitter and beam divergence angle. 
- Each region with radius $r_{j}$ is divided into subregions for each AV with the index $i \in[1, N]$ such that the channel response is not time-varying in those durations with radius $r_{j, i} \equiv \vec{v}_{i} T_{j} / 2$ where $T_{j}$ is the coherence time of the channel in the $j$ th region and $\vec{v}_{i}$ is the velocity of the $i$ th vehicle. It is assumed that AV has a constant velocity throughout the overall duration of the coding in the $j$ th region and AVs pass through the center of each region for simplicity. Then there is a set of $k_{j, i} \equiv r_{j} / r_{j, i}$ different subregions for the $i$ th vehicle. $\Delta t_{j, i} \equiv 2 r_{j} / \vec{v}_{i}=k_{j, i} T_{j}$ denotes the duration for which the $i$ th vehicle passes through the $j$ th region for some integer $k_{j, i}$. Assume that $k_{j}^{\max }$ is the largest one in the set $k_{j, i}$ for $i \in[1, N]$ corresponding to the slowest plane.

- Each vehicle passes through an ordered subset of $M$ regions denoted by $\operatorname{Reg}_{i} \subseteq[1, M]$ such that some vehicle passes through a smaller number of regions in the STMC coding area.

- It is assumed that different vehicles can pass through the same regions at similar or different times. Therefore, the coding is optimized to satisfy the demands of the users based on the coarse knowledge of the paths with $R e g_{i}$. If $r_{j}$ is large enough, then there can be a large number of AVs with the same altitude in the same region while AVs with different but close values of altitudes can fly in the same region with a small radius.

- For simplicity, assume that the receiver characteristics are the same for each $i$ th aerial vehicle creating identical capacity channels for the time interval $\left[(k-1) T_{j}, k T_{j}\right]$ with the same duration $T_{j}$ and channel properties for $j$ th region corresponding to the time period with the index $k_{j, i}=k$.

- Total duration of multireceiver STMC data transmission is determined by the rough knowledge of the timing for the vehicles passing through the coding area. The knowledge is obtained either by uplink communications between the vehicles and the transmitter source or by radar target tracking approaches. Therefore, the coded stream in the $j$ th region for the duration of $k_{j}^{\max } \times T_{j}$ is repeated for $l$ times for the vehicle to pass through the region. The repeat procedure functions as a form of spatial wireless caching for AVs while it is minimized with respect to the knowledge about the topology of the network.

- Each region with index $j$ is assumed to have a channel capacity of $C_{j, m}$ bits/period for $m \in\left[1, k_{j}^{\max } \times l\right]$ covering all $k_{j}^{\max } \times l$ coherence period durations. Furthermore, assume that $C_{j, m}=U_{j, m} n_{j} C_{j, m, u}$, where $U_{j, m}$ is the number of coded transmissions, $n_{j}$ is the transmission symbol block length assigned to the $j$ th region, and $C_{j, m, u}$ is the bits per channel use for each coded transmission with the index $u$.

Then STMC for multiple aerial vehicles is defined formally as follows, where the path information and the channel state information for the regions are utilized for a global encoding transmission architecture:

Definition 1 STMC for downlink network of multiple AVs: Message $W_{i}$ is uniformly distributed over the following set:

$$
\mathcal{W}_{i}:\left\{1,2, \ldots 2^{F_{i}}\right\},
$$

where $F_{i}$ is the bit size of messages or files. A global encoding function $E_{j, m, u}$ for each region with the index $j$, period index $m$, and coded transmission index $u$ encodes the transmission data as follows:

$$
E_{j, m, u}: \mathcal{W}_{1} \times \ldots \times \mathcal{W}_{N} \rightarrow \mathcal{X}_{j, m, u}
$$


where the size of the message set $\mathcal{W}_{1} \times \ldots \times \mathcal{W}_{N}$ is given by $\prod_{i=1}^{N} 2^{F_{i}}$, the coded symbol set $\mathcal{X}_{j, m, u}$ has the size $2^{n_{j} C_{j, m, u}}, j \in[1, M], m \in\left[1, k_{j}^{\max } \times l\right]$, and $u \in\left[1, U_{j, m}\right]$. Then the $i$ th aerial vehicle decodes the received data symbol set with the decoding function $D_{i}$ such that the $i$ th symbol is estimated as follows:

$$
D_{i}: \prod_{j \in R e g_{i}} \prod_{m=1}^{k_{j, i}} \prod_{u=1}^{U_{j, m}} \mathcal{X}_{j, m, u} \rightarrow \widehat{\mathcal{W}}_{i}
$$

where the $\prod$ operator denotes the tensor product operation for the sets and $\widehat{W}_{i}$ is the estimated message for the ith vehicle.

The explicit design of coding and performance analysis are left as future works, while the problem formulation for encoding and decoding of data in STMC is provided in Definition 1. It provides the most general definition of coding by considering collective paths of all the planes in the target region throughout the time duration determined with respect to the slowest planes to complete their tracks along the process of decoding.

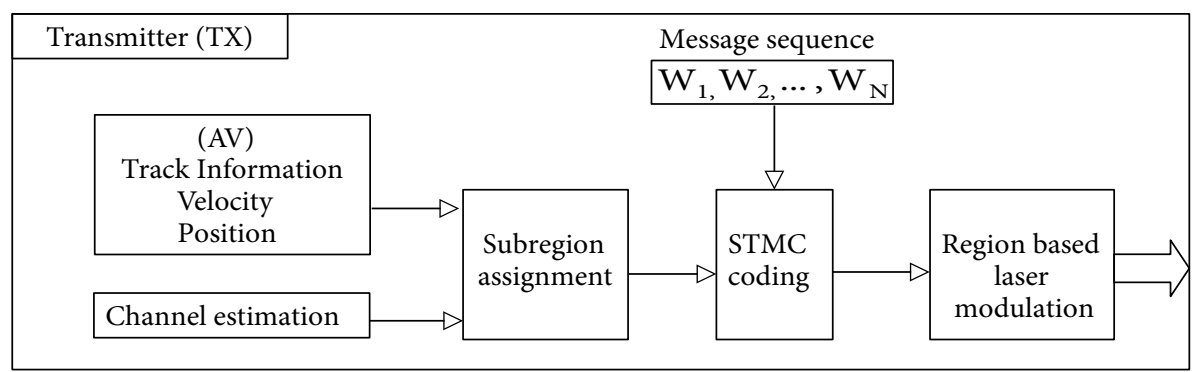

(a)

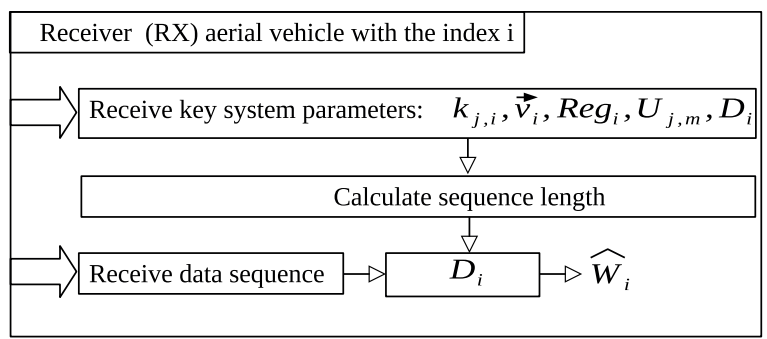

(b)

Figure 4. (a) STMC transmitter modulation/encoding design, where vehicle related coarse tracking data and channel state are estimated before the data transmission. (b) Receiver encoding block diagram, where key parameters and decoding function are received in addition to the data sequence.

Algorithm 1 summarizes the fundamental building blocks of the STMC coding shown in Figure 4 and described in Definition 1, while Algorithm 2 summarizes the decoding algorithm. The transmitter and receiver block diagrams are shown in Figures $4 \mathrm{a}$ and $4 \mathrm{~b}$, respectively. The encoder in the satellite, as presented in Algorithm 1, coarsely tracks the positions and velocities $\left(\vec{v}_{i}\right)$ of the planes with their respective routes. It forms a sequential map of the planes throughout the duration of data transmission. Then the requested message sequences $W_{i}$ for each plane with the index $i$ for $i \in[1, N]$ are obtained by using the uplink channel with the planes. The divergence angle $\theta_{j}$ is either preset or optimized based on the current sequential maps of the planes. Then the respective regions are calculated and determined with the number, position, and diameter 


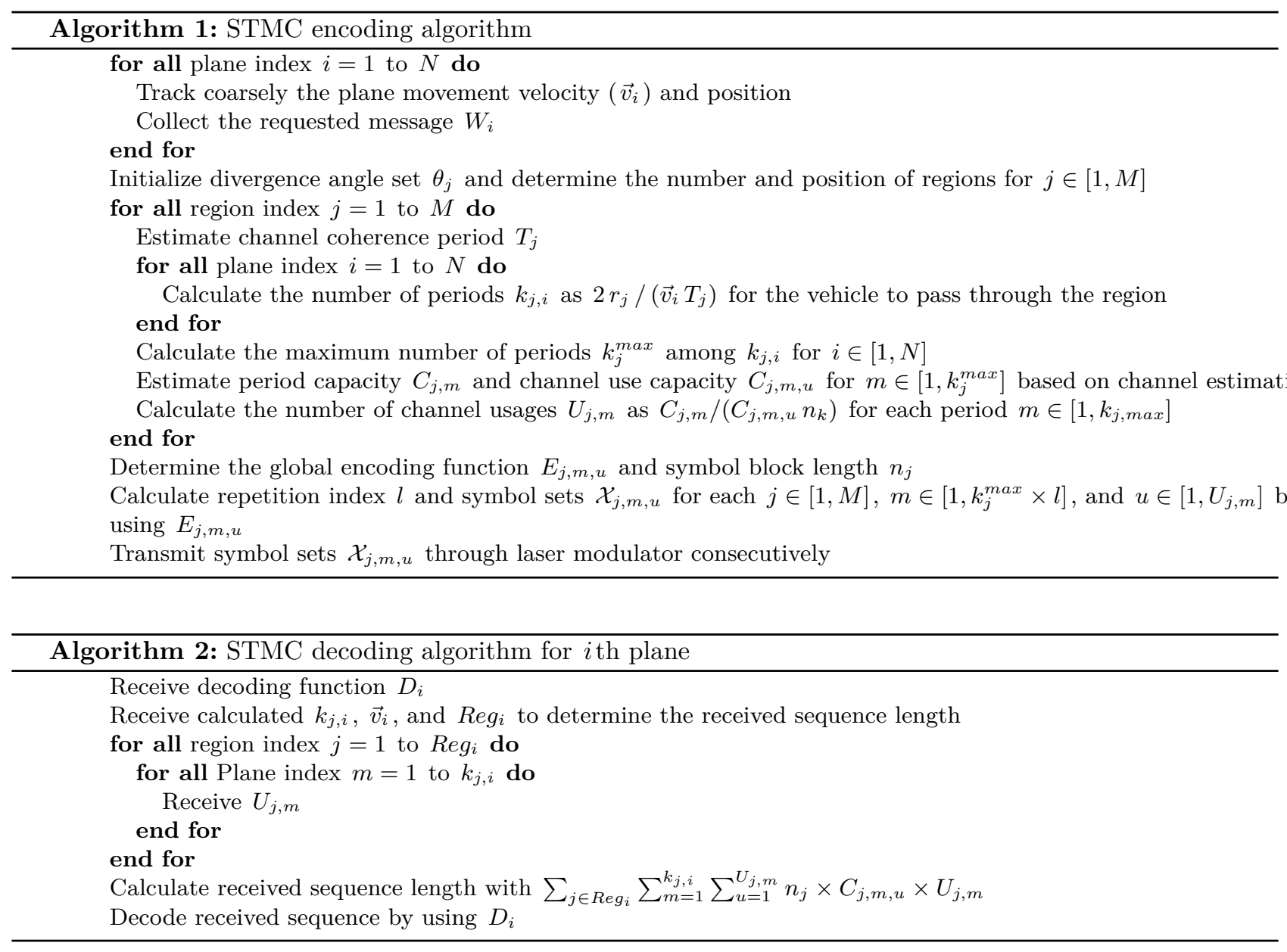

$r_{j}$ for $j \in[1, M]$. For each region, the channel fading and coherence period $T_{j}$ are estimated. The number of periods $k_{j, i}$ for each $j$ th region and $i$ th plane is calculated for the vehicle to pass through the region. Their maximum is calculated as $k_{j}^{\max }$. Then, based on the fading and channel path loss information, channel capacities for each period $T_{j}$ and for each channel use are calculated as $C_{j, m}$ and $C_{j, m, u}$, respectively, for $j \in[1, M]$ and $m \in\left[1, k_{j}^{\max }\right]$. The number of channel usages $U_{j, m}$ for each period is calculated. Then the optimum encoding function $E_{j, m, u}$ mapping a specific message set to the symbol sequence $X_{j, m, u}$ for each $j \in[1, M], m \in\left[1, k_{j}^{\max } \times l\right]$ and $u \in\left[1, U_{j, m}\right]$ among the symbol set $\mathcal{X}_{j, m, u}$ is calculated based on the sequential mapping of the planes, the properties of the regions, their capacities, and the requested message sets from the planes. Then each symbol $X_{j, m, u}$ is transmitted consecutively to the planes.

On the other hand, in the decoder, as shown in Algorithm 2, the key system parameters $k_{j, i}, \vec{v}_{i}, \operatorname{Reg}_{i}$, and $U_{j, m}$ are received before the data transmission begins to calculate the length of the transmitted sequence. Similarly, designed decoder function $D_{i}$ in the transmitter is received before the transmission begins. The encoder and decoder functions can also be designed and set in the memories of the transmitter and receiver. In this article, the most general definition of STMC is introduced, covering all possible mechanisms to transmit downlink data.

STMC coding for dependent messages such as file requests including the same packets or messages, such 
as downloading a popular multimedia video, results in wireless caching for aerial networks. It is possible to define the optimization problem for both independent messages and caching architectures by utilizing the available formulations, such as index coding, content caching, and coded broadcast algorithms [10, 11]. For example, in a drone network, tactical information or some broadcast control data are spatially cached on specific regions along the routes of the drones. The problem definitions and solutions for STMC multiple vehicle coding and spatio-optical caching are left as future works. On the other hand, for STM regions with large $r_{j}$ values, there is a significant number of AVs in the same region, making each coded transmission be received by multiple AVs at the same time. The STMC architecture includes the broadcast mechanism discussed previously for VLC channels as a special case in the global encoding operation [26].

\section{Numerical simulations and discussions}

STMC design parameters are simulated to analyze the effects of beam divergence angle on the STM region radius, time duration for AVs passing through each region, and capacity for each coded transmission in that region. In Figure 5, laser beam divergence angle $\theta$ is simulated for varying aperture size $D_{T}$ and wavelength $\lambda$, showing that operating with large beam angle allows smaller transmitter aperture size and weight as another advantage of STMC system design. On the other hand, more regions are created with multiple apertures in the same area on the transmitter.

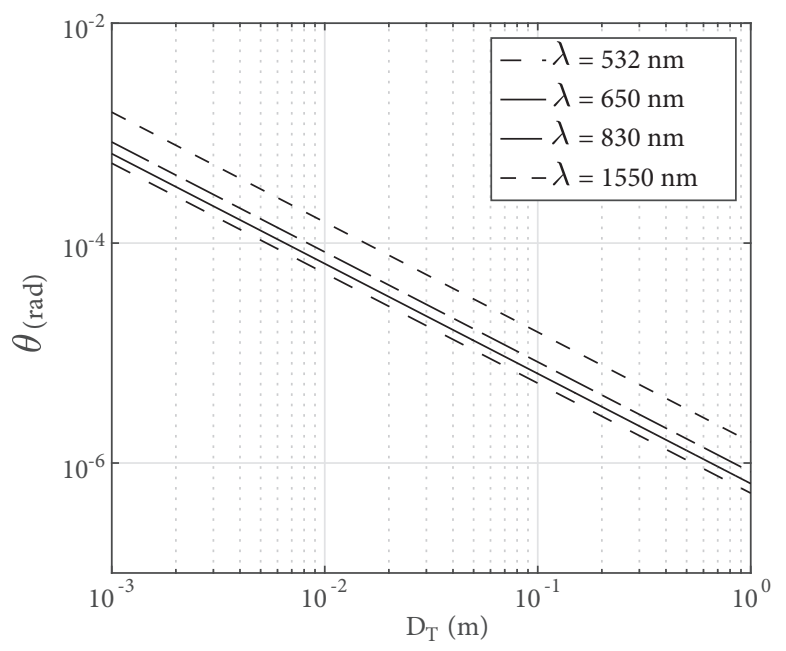

Figure 5. Laser beam divergence angle $\theta$ for varying aperture size $D_{T}$ and wavelength $\lambda$.

It is assumed that an ordinary drone has an area with diameter less than $D_{A v}=1 \mathrm{~m}$ while a plane has less than $100 \mathrm{~m}$. Then the STM regions are denoted as the regions where $r_{j}$ is large enough to include multiple AVs at the same time without requiring accurate tracking and providing a broadcast region. In Figure $6 \mathrm{a}, r_{j}$ for varying distance $L$ to the receiver and for varying $\theta_{j}$ are shown. The STM region for drones starts at $r_{j}=1 \mathrm{~m}$ while for larger AVs such as planes it is starting from $r_{j}=100 \mathrm{~m} . r_{j}$ increases with both increasing $L$ and $\theta_{j}$. In Figure $6 \mathrm{~b}$, the duration for the $i$ th vehicle with velocity $\vec{v}_{i}$ to pass through region $r_{j}$, i.e. $\Delta t_{j, i}$, is shown for varying $\mathrm{AV}$ velocities including slow vehicles such as drones and high velocities including airplanes. It is observed that the duration reaches values between tens and thousands of seconds, which are much larger than coded transmission interval $T_{j}$, i.e. $0.1-10 \mathrm{~ms}$ [4]. This provides a broadcast region with various advantages such as allowing time diversity, providing STMC global encoding mechanisms for multiple AVs in the same area, 
and serving significant numbers of AVs simultaneously while decreasing the effects of lower data rates due to larger beam divergence angle.

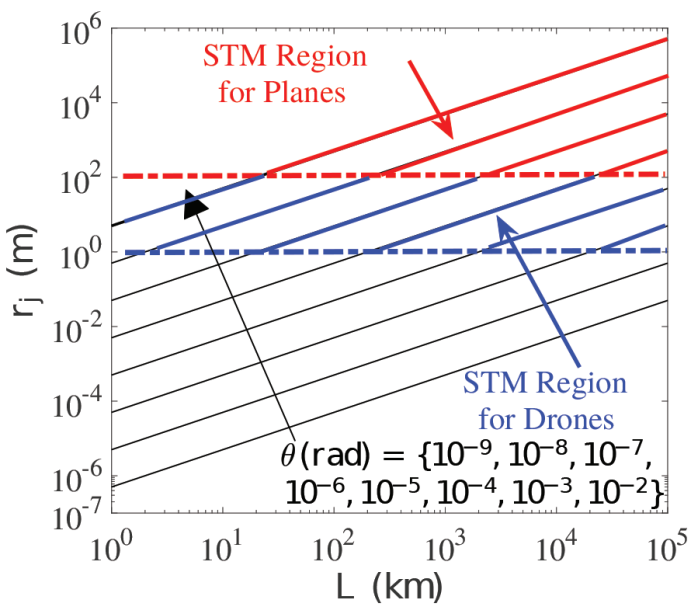

(a)

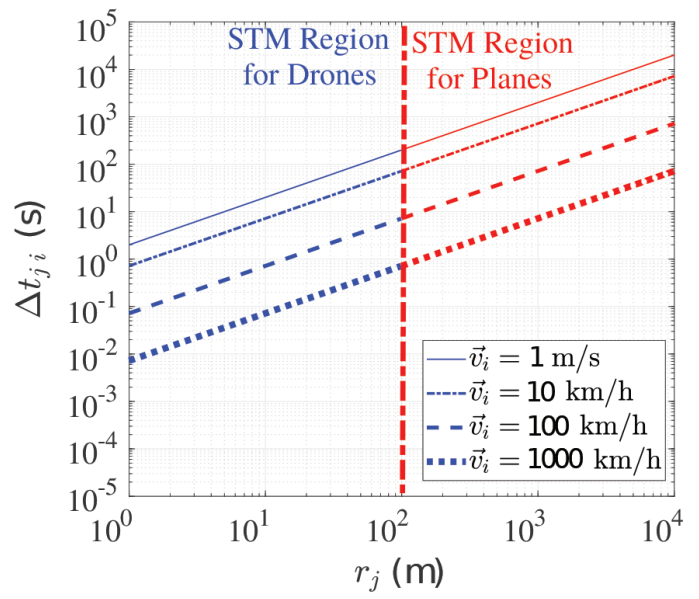

(b)

Figure 6. (a) STM region radius values for varying distance $L$ to the receiver and varying $\theta$ where STM regime is assumed to be $r_{j}>D_{A v}$ for planes with the assumption $D_{A v} \approx 100 \mathrm{~m}$ for planes and $D_{A v} \approx 1 \mathrm{~m}$ for drones, and (b) $\Delta t_{j, i}$ for varying $\mathrm{AV}$ velocity $\vec{v}_{i}$ and $\mathrm{STM}$ radius $r_{j}$.

\subsection{GEO broadcasting scenario for planes}

In satellite FSO links including GEO and LEO ranges, beam divergence angles in the range between several $\mu \mathrm{rad}$ and mrad are utilized [3, 21]. Assume that parameters in the Table are utilized based on the experimental verification in [22] for a simple scenario of GEO broadcasting onto the swarm of planes as shown in Figure 7. On the other hand, data rates between tens of Mbit/s and several Gbit/s are achieved with tens of watts transmission power and tens of centimeters aperture sizes. In [21], a downlink channel with $20 \mu \mathrm{rad}$ beam divergence angle and $830 \mathrm{~nm}$ wavelength performs $\approx 1 \mathrm{Mbit} / \mathrm{s}$ data rates between a satellite at an altitude of $2286 \mathrm{~km}$ and a ground station with $13 \mathrm{~W}$ transmission power. Similarly, in [22], an optical bandwidth of $B_{o}=19 \mathrm{GHz}$ and electrical bandwidth of $B_{e}=0.9 \mathrm{GHz}$ with return to zero (RZ) coding, aperture diameter of $D_{T}=0.135 \mathrm{~m}$, transmit power of $40 \mathrm{dBm}$, and $\lambda=1550 \mathrm{~nm}$ achieves $1 \mathrm{Gbit} / \mathrm{s}$ data rates with BER of $10^{-9}$, received power of $-50.3 \mathrm{dBm}$, and link margin of $9.7 \mathrm{~dB}$ between a HAP and a GEO satellite at altitudes of 20 $\mathrm{km}$ and $35786 \mathrm{~km}$, respectively. The reference divergence angle for the proposed set-up gives $\theta_{0} \approx 11.5 \mu \mathrm{rad}$. Furthermore, assume that the reference distance is $L_{0} \approx 35750 \mathrm{~km}$. The channel models are analyzed in detail in the literature in terms of both experimental and numerical simulation studies [3, 4].

A simplified model allows to simulate the effect of $\theta_{j}$ on the trade-off between STM region radius $r_{j}$ and data rate in the links on that region. Assuming the on-off keying (OOK) modulation in [22] satisfies a bit-error-rate $(\mathrm{BER})$ of $Q(\sqrt{S N R / 2})=10^{-9}$ [27] resulting in the minimum SNR of $\approx 10.7831 \mathrm{~dB}$ with the minimum received power of $P_{R, 0} \approx 10 \mathrm{nW}$ based on $-50.3 \mathrm{dBm}$ sensitivity and noise power of $P_{N_{0}} \approx 0.139 \mathrm{nW}$. $Q($.$) denotes the q-function Q(x) \equiv(1 / \sqrt{2 \pi}) \int_{x}^{\infty} e^{-u^{2} / 2} d u$. Noise spectral sensitivity of $N_{0} \equiv 0.139 / B_{e} \approx$ $1.5410^{-19} \mathrm{~W} / \mathrm{Hz}$ is assumed. Then it is assumed that received power for a specific STM divergence angle $\theta$ is given as follows: 
Table. Numerical simulation parameters of GEO broadcasting scenario for planes.

\begin{tabular}{|l|l|l|}
\hline Parameter & Symbol & Values \\
\hline Divergence angle & $\theta_{j}$ & $10^{-6}$ to $10^{-1}(\mathrm{rad})$ \\
\hline Reference received power & $P_{R, 0}$ & $10(\mathrm{nW})$ \\
\hline Noise power & $N_{0}$ & $1.5410^{-19}(\mathrm{~W} / \mathrm{Hz})$ \\
\hline Optical bandwidth & $B_{o}$ & $19(\mathrm{GHz})$ \\
\hline Electrical bandwidth & $B_{e}$ & $0.9(\mathrm{GHz})$ \\
\hline Rate & $R_{c}$ & $10^{3}, 10^{5}, 10^{7}, 10^{9}(\mathrm{bit} / \mathrm{s})$ \\
\hline Reference distance & $L_{0}$ & $35750(\mathrm{~m})$ \\
\hline Wavelength & $\lambda$ & $1550(\mathrm{~nm})$ \\
\hline
\end{tabular}

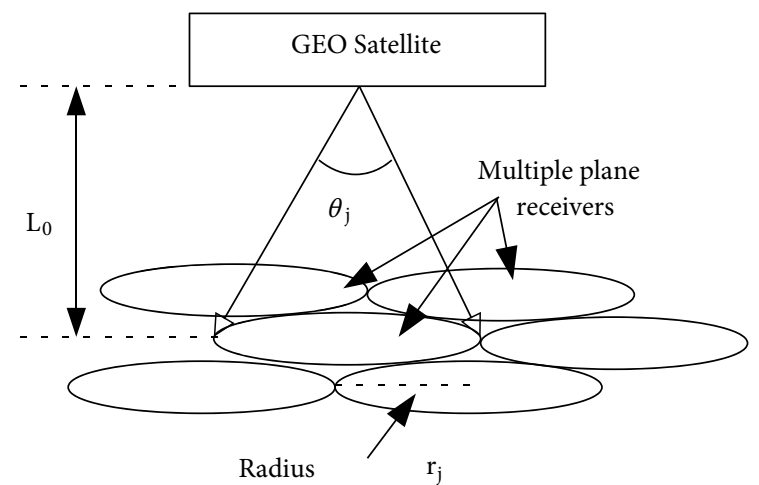

Figure 7. GEO satellite broadcasting scenario for the planes where the parameters in the Table are utilized.

$$
P_{R}(\theta)=P_{R, 0} \frac{\theta_{0}^{2}}{\theta^{2}}
$$

Then the effect of increasing $r_{j}$ on capacity for each coded transmission interval $T_{j} / U_{j, m}$ is modeled with $C(\theta)=B_{e} \log \left(1+P_{R}(\theta) / P_{N_{0}}\right.$ ) (without utilizing time divergence along the track of AV) while it is numerically simulated as shown in Figure 8a. The bandwidth efficiency in terms of bit $/ \mathrm{s} / \mathrm{Hz}$ drops significantly as shown in Figure $8 \mathrm{~b}$ to less than $1 \mathrm{bit} / \mathrm{s} / \mathrm{Hz}$ as $r_{j}$ increases. However, increasing $r_{j}$ to values of $1000 \mathrm{~km}$ still allows data communication capability of tens of bit/s while broadcasting a significant number of airplanes or AVs in the same area, providing simultaneous coding of the requested data to be shared by each AV. On the other hand, compared with the more complicated coding mechanisms described in Definition 1, time diversity by simple repetitive coding improves the data rate significantly. As $r_{j}$ gets larger, duration $\Delta t_{j, i}$ for each AV increases by allowing more complicated coding mechanisms in that region compared with the narrow beam and high data rate links with small $r_{j}$.

BER probability is approximately calculated by $Q(\sqrt{S N R / 2})$ by assuming OOK modulation where SNR is calculated with $P_{R}(\theta) /\left(R_{c} N_{0}\right)$ by utilizing data rate $R_{c}$ and bandwidth dependent noise power. It is observed in Figure $8 \mathrm{c}$ that as data rate $R_{c}$ increases, the reliable communication radius $r_{j}$ and divergence angle $\theta_{j}$ decrease. The decrease in data rate increases $r_{j}$ while not requiring precise tracking and serving simultaneously multiple AVs in the same area determined by $r_{j}$. In Figures $8 \mathrm{~d}-8 \mathrm{f}$, the effects of varying $\theta_{j}$ on BER and SNR values simulated in Figure $8 \mathrm{c}$ are shown for $R_{c}$ being $10^{3}$, $10^{5}$, and $10^{7}$ bit/s, respectively. It is observed that decreasing data reduce the effective noise power and increase both $r_{j}$ and $\theta_{j}$, allowing a 
higher broadcast region and simultaneous data transmission to multiple AVs. Observe that the proposed data rates are much smaller than the Gbit/s data rates with accurate tracking. The proposed system design presents a trade-off between hardware complexity and data rate while serving multiple AVs simultaneously as a highly important novelty. In other words, the decrease in data rate per AV is balanced by an increase in the number of multiple AVs receiving data and the decrease in the hardware complexity.

\section{Challenges and open issues}

There is a set of challenges and future works for the designed STMC system to be optimized, listed as follows:

- The channel effects, e.g., absorption, scattering loss, turbulence, beam wandering loss, ambient light, and

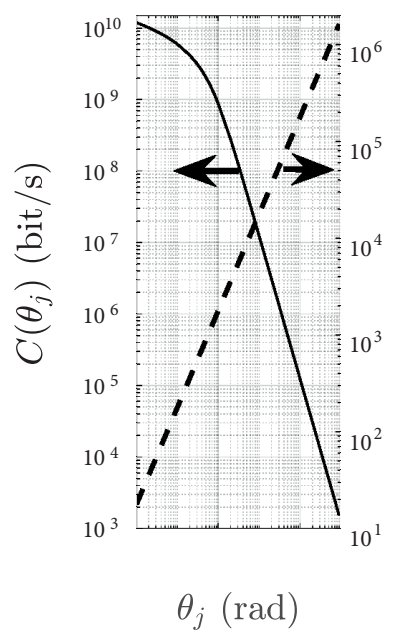

(a)

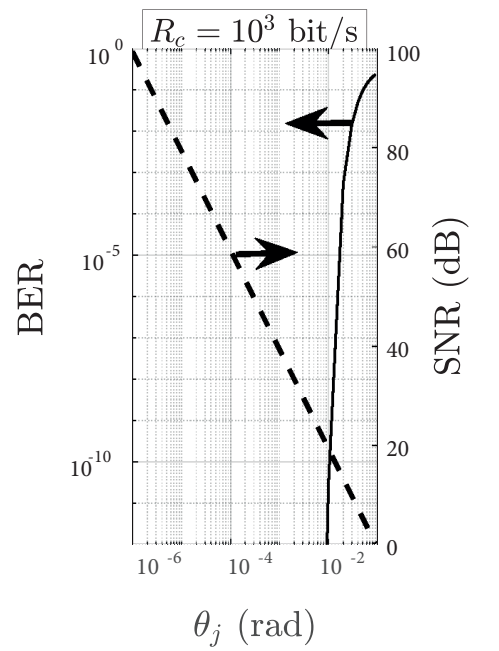

(d)

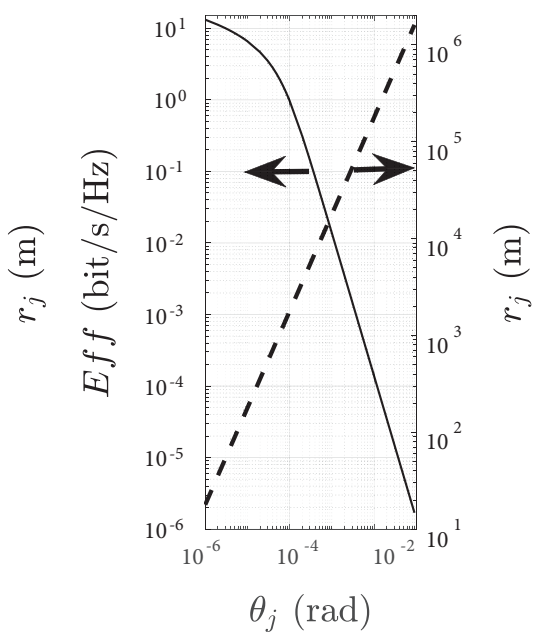

(b)

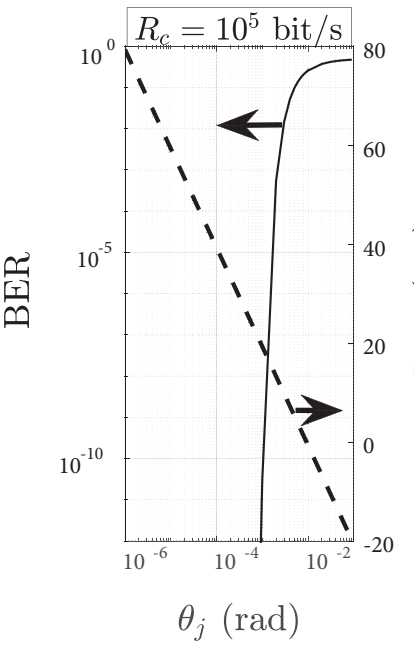

(e)

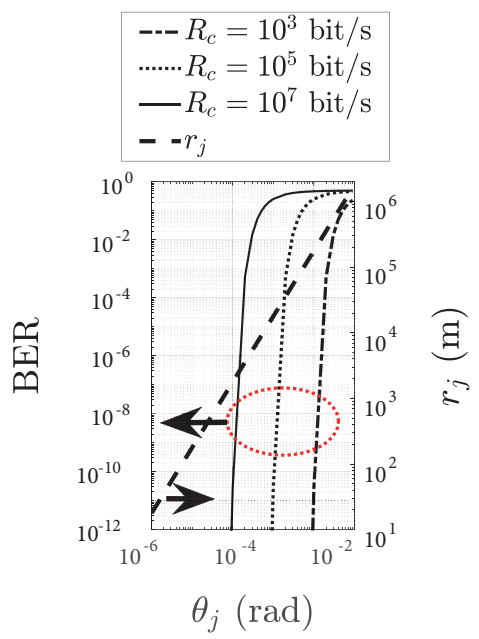

(c)

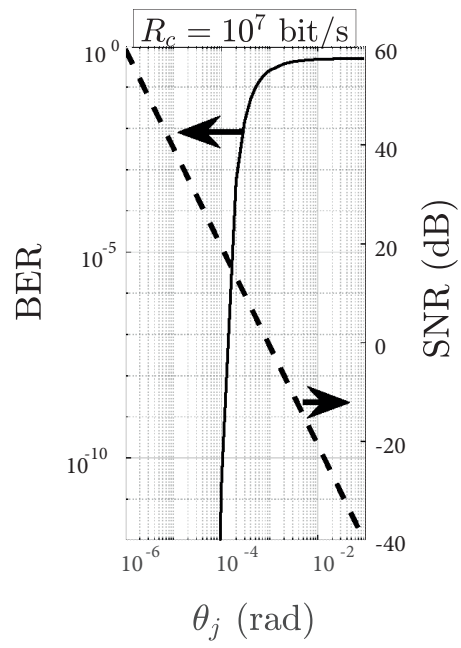

(f)

Figure 8. (a) Capacity $C_{j, m, u}$ and denoted by $C(\theta)$ and (b) bandwidth efficiency (Eff) in $u$ th coded transmission in the time interval $\left[(m-1) T_{j}, m T_{j}\right]$ in the region with radius $r_{j}$ for varying laser beam divergence angle $\theta_{j}$ for the simulation parameters in the Table. (c) BER for varying data rate $R_{c}$ of $10^{3}, 10^{5}$, and $10^{7}$ bit/s (d) in the region with radius $r_{j}$ for varying $\theta_{j}$. BER and SNR for varying $\theta_{j}$ where $R_{c}$ is equal to (d) $10^{3}$, (e) $10^{5}$, and (f) $10^{7} \mathrm{bit} / \mathrm{s}$. 
background interference, for both long range satellite communications and short range drone communications should be modeled in detail for optimum STMC system encoding purposes by analyzing both VLC and FSO system architectures. The effects on $T_{j}$, delay time for the downlink channel, and the velocity of high speed AVs should be theoretically and experimentally modeled [4].

- The uplink mechanism should be synchronized for specific bidirectional applications. Broadcast applications are future promising works, such as spatio-optical caching of multimedia contents, control data transmission, and applications similar to GPS.

- The STMC design in Definition 1 gives an opportunity as a future work to define a global encoding problem for specific applications such as spatio-optical caching and broadcasting [10, 11].

\section{Conclusions}

In this article, a novel modulation and coding mechanism denoted by STMC is proposed for crowded downlink networks of AVs. It does not require high accuracy tracking, synchronization, or costly FSO components in high gain satellite and AV hardware platforms. STMC defined for multiuser networks of AVs promises a rich set of applications with flexible design capability for varying altitudes and velocities of transmitters and receivers. It achieves multiuser data transmission without complicated hardware such as high accuracy tracking and synchronization, narrow beam laser sources, and high gain costly receiver circuitry designed for high data rate communications. Thousands of kilometers of coverage area is achieved with tens of watts reference transmitter power allowing data communication capability of tens of bit/s while broadcasting to significantly larger numbers of airplanes simultaneously. The STMC problem is information theoretically defined and modeled. STMC fuses information regarding tracks with geographical and positional data implementing novel broadcast applications similar to GPS. In addition, spatio-optical wireless caching mechanism is, for the first time, introduced for broadcast based cooperation between AVs and transmission of the same information content such as popular multimedia files. Future works and challenging open issues have been discussed.

\section{Acknowledgments}

This work was supported by Vestel Electronics, Manisa, Turkey, and the author would like to thank Dr Görkem Memişoğlu for fruitful discussions.

\section{References}

[1] Fidler F, Knapek M, Horwath J, Leeb WR. Optical communications for high-altitude platforms. IEEE J Sel Top Quant 2010; 16: 1058-1070.

[2] Motlagh NH, Taleb T, Arouk O. Low-altitude unmanned aerial vehicles-based internet of things services: comprehensive survey and future perspectives. IEEE Internet Things J 2016; 3: 899-922.

[3] Kaushal H, Kaddoum G. Optical communication in space: challenges and mitigation techniques. IEEE Commun Surv Tut 2017; 19: 57-96.

[4] Kaushal H, Kaddoum G. Free space optical communication: challenges and mitigation techniques. ArXiv preprint 2015; 1506.04836 .

[5] Gulbahar B, Sencan S. Wireless Internet service providing for 5G with hybrid TV broadcast and visible light communications. In: IEEE/IFIP 2017 Wireless Days Conference; Porto, Portugal; 2017. pp. 66-69. 
[6] Cazaubiel V, Planche G, Chorvalli V, Le Hors L, Roy B, Giraud E, Lidovic V, Carre F, Decourbey E. LOLA: A $40.000 \mathrm{~km}$ optical link between an aircraft and a geostationary satellite. In: SPIE 2006 International Conference on Space Optics; Noordwijk, the Netherlands; 2006. pp. 10567261-10567267.

[7] Gulbahar B, Memisoglu G. Communication system and communication method. EPO European Patent Application 2017; EP3407509A1.

[8] Rahman MA. Enabling drone communications with WiMAX technology. In: IEEE 2014 5th International Conference on Information, Intelligence, Systems and Applications; Chania, Greece; 2014. pp. 323-328.

[9] Skinnemoen H. UAV and satellite communications live mission-critical visual data. In: IEEE 2014 International Conference on Aerospace Electronics and Remote Sensing Technology ICARES; Yogyakarta, Indonesia; 2014. pp. 12-19.

[10] Ji M, Caire G, Molisch AF. Fundamental limits of caching in wireless D2D networks. IEEE T Inf Theory 2016; 62: 849-869.

[11] Bar-Yossef Z, Birk Y, Jayram TS, Kol T. Index coding with side information. IEEE T Inf Theory 2011; 57: 14791494.

[12] Graham-Rowe D. Solar-powered lasers. Nat Photonics 2010; 4: 64-65.

[13] Phipps CR, Bonnal C. A spaceborne, pulsed UV laser system for re-entering or nudging LEO debris, and re-orbiting GEO debris. Acta Astronaut 2016; 118: 224-236.

[14] Guan Z, Zhao CM, Yang SH, Wang Y, Ke JY, Zhang HY. Demonstration of a free-space optical communication system using a solar-pumped laser as signal transmitter. Laser Phys Lett 2017; 14: 055804.

[15] Majumdar AK, Ricklin JC. Free-space Laser Communications: Principles and Advances. New York, NY, USA: Springer Science Business Media, 2010.

[16] Aveta F, Refai HH, LoPresti P. Multi-user FSO communication link. In: IEEE 2017 Cognitive Communications for Aerospace Applications Workshop; Cleveland, OH, USA; 2017. pp. 1-5.

[17] Riza NA. Reconfigurable optical wireless. In: IEEE 1999 12th Annual Meeting of Lasers and Electro-Optics Society; San Fransisco, CA, USA; 1999. pp. 70-71.

[18] Grant MA, Robson D, Matthews NF. Multiple access communication system. United States Patent 1992; US 5119225.

[19] Shen TC, Drost RJ, Davis CC, Sadler BM. Design of dual-link (wide-and narrow-beam) LED communication systems. Opt Express 2014; 22: 11107-11118.

[20] Grobe L, Paraskevopoulos A, Hilt J, Schulz D, Lassak F, Hartlieb F, Kottke C, Jungnickel V, Langer KD. High-speed visible light communication systems. IEEE Commun Mag 2013; 51: 60-66.

[21] Wilson KE. An overview of the GOLD experiment between the ETS-VI satellite and the Table Mountain facility. Telecommunications and Data Acquisition Progress Reports 1995; 42: 8-19.

[22] Fidler F. Optical backhaul links between HAPs and satellites in the multi-Gigabit regime. In: IEEE 2008 GLOBECOM Workshops; New Orleans, LA, USA; 2008. pp. 1-5.

[23] Kaushal H, Jain VK, Kar S. Free Space Optical Communication. New York, NY, USA: Springer, 2017.

[24] Fidler F. Optical communications from high-altitude platforms. PhD, Vienna University of Technology, Vienna, Austria, 2007.

[25] Klein BJ, Degnan JJ. Optical antenna gain. 1: Transmitting antennas. Appl Opt 1974; 13: $2134-2141$.

[26] Ma S, Yang R, Li H, Dong ZL, Gu H, Li S. Achievable rate with closed-form for SISO channel and broadcast channel in visible light communication networks. J Lightwave Technol 2017; 35: 2778-2787.

[27] Proakis JG. Digital Communications. New York, NY, USA: McGraw-Hill, 1995. 